\title{
Magneto-optical Stark effect in Fe-doped CdS nanocrystals
}

\author{
Mahima Makkar, ${ }^{\dagger \nabla}{ }^{2}$ Lakshay Dheer, ${ }^{\text {\#* }}$ Anjali Singh, ${ }^{\complement}$ Luca Moretti, ${ }^{f}$ Margherita Maiuri, ${ }^{f}$ \\ Soumen Ghosh, ${ }^{f}$ Giulio Cerullo, ${ }^{f}$ Umesh V Waghmare, ${ }^{\dagger \nabla}$ Ranjani Viswanatha ${ }^{\dagger \nabla} \Delta^{*}$
}

${ }^{\dagger}$ New Chemistry Unit, "Theoretical Sciences Unit, "Chemistry and Physics of Materials Unit, ${ }^{\Delta}$ International Centre for Materials Science, ${ }^{\nabla}$ School of Advanced Materials, Jawaharlal Nehru Centre for Advanced Scientific Research, Jakkur, P.O., Jakkur, Bangalore 560064, India.

'Center for Study of Science, Technology and Policy, Bangalore 560094, India.

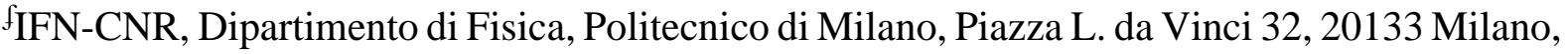
Italy.

e equal contribution

\begin{abstract}
$\mathrm{Fe}^{2+}$ doping in II-VI semiconductors, due to the absence of energetically accessible multiple spin state configurations, has not given rise to interesting spintronic applications. In this work, we demonstrate for the first time that the interaction of homogeneously doped $\mathrm{Fe}^{2+}$ ions with the host CdS nanocrystal with no clustering is different for the two spin states and produces two magnetically inequivalent excitonic states upon optical perturbation. We combine ultrafast transient absorption spectroscopy and density functional theoretical analysis within the ground and excited states to demonstrate the presence of the magneto-optical Stark effect (MOSE). The energy gap between the spin states arising due to MOSE does not decay within the time frame of observation, unlike optical and electrical Stark shifts. This demonstration provides a stepping-stone for spin-dependent applications.
\end{abstract}

Keywords: Stark effect, magneto-optics, spintronics, Fe doping, luminescence quenching

The combination of nanotechnologies with quantum physics uses the power of laws of physics for practical purposes. The use of the spin degree of freedom of an electron, specifically in a system with strong spin-orbit coupling leading to ultrafast spin selectivity, ${ }^{1}$ promises to lead to enhancement of data processing speed and integration densities, allowing a reduction in electrical power consumption in comparison to the conventional electronic devices. ${ }^{2-4}$ However, the most important parameter for potential applications in spintronics is the presence of multiple low energy spin configurations of the transition metal ions. Hence, even though iron is naturally magnetic, so far there has been no demonstration of spintronic-like states in $\mathrm{Fe}^{2+}$ doped simple II-VI semiconductors. ${ }^{5,} 6$ However, the spin of the Fe dopant is very sensitive to the local environment and CdS nanocrystals (NCs) have the potential to strongly hybridize with magnetic ions, beyond the classical description of diluted magnetic semiconductors. ${ }^{5,7}$ Until now, however, the power of spatial confinement of the wavefunction as well as the role of dopant-host interactions, free of dopant-dopant interactions, has not been explored. Here we combine ultrafast transient absorption spectroscopy and density functional theory within the ground and excited states to demonstrate that the uneven interaction of the 
two spin states for uniformly doped $\mathrm{Fe}^{2+}$ ions in spatially confined $\mathrm{CdS}$ NCs produces two magnetically inequivalent excitonic states upon mild optical perturbation. Interestingly, this energy gap arises from a newly discovered phenomenon that we label as Magneto-Optical Stark Effect (MOSE) and does not decay within the time frame of observation. Further, this gap corresponds to $\sim 2 \mathrm{~T}$ external magnetic field that would be required to obtain this splitting from the Zeeman effect at room temperature, thus demonstrating a long-lived splitting.

Modulation of the absorption edge of semiconductors with external perturbations like an applied electric field ${ }^{8,9}$ or an optical pulse, ${ }^{1,10}$ leads to transient shifts in energy within the bandgap region, due to the non-linear interaction of electronic states with the electric field/photons, and is known as the Stark shift. In a system wherein the quantum states are strongly coupled to phonons through exciton-phonon interactions, the optical Stark effect (OSE) can be used to generate closely spaced spin selective states. However, most often the quantum states affected by OSE are buried deep in the continuum ${ }^{11}$ and OSE is only present for the duration of the illuminating light pulse, typically on ultrafast (femtosecond) timescales ${ }^{12,13}$ and are hence not very practical for use in device applications. Additionally, the possibility of impact ionization ${ }^{14}$ due to the intense external stimulus limits the modulation of the absorption edge through the Stark effect. However, the observation of an enhanced Stark effect in quantum-confined materials compared to their bulk counterparts significantly enhances their chances in real applications. ${ }^{15,16}$

In this work, we demonstrate the power of small optical perturbations in presence of a $\mathrm{Fe}^{2+}$ dopant uniformly distributed in a semiconductor to generate long-lived spin-selective excited states using the example of $\mathrm{Fe}^{2+}$ doped CdS NCs. We observe that the interaction of $\mathrm{Fe}^{2+}$ acceptor states, uniformly doped within the CdS host semiconductor, is strong and interestingly spin selective. Upon excitation with a weak optical pulse, we demonstrate that the spin-down transition interacts strongly with the host while the spin-up states are largely unaffected, as clearly observed from the DFT calculations, leading to a spin splitting of the degenerate excitonic states in $\mathrm{Fe}^{2+}$ doped $\mathrm{CdS}$ systems. In contrast to OSE, this effect is long-lived and stronger than the best reported shifts so far at room temperature, using a weak excitation fluence of $24 \mu \mathrm{J} / \mathrm{cm}^{2}$ (Table S1, Supporting information (SI)). We term this phenomenon as MOSE and establish it with a combination of ultrafast transient absorption (TA) spectroscopy and theoretical analysis of ground and excited states.

It is important to note here that uniformity of the $\mathrm{Fe}^{2+}$ doping in the NCs is crucial to observe interesting magnetic phenomena. ${ }^{17-19}$ Doping in NCs with uniform distribution of the dopants in the bulk rather than the surface is plagued with non-trivial problems of phase segregation, leading to the non-uniform distribution of magnetic dopants. However, recent literature has shown that the use of diffusion of the dopants inside out is successful in generating uniform doping of $\mathrm{Fe}^{2+}$ in the NCs. ${ }^{20,21}$ Here we use a similar technique to obtain $\mathrm{Fe}^{2+}$ doped CdS NCs wherein $\mathrm{Fe}_{3} \mathrm{O}_{4}$ was overcoated with a thick shell of $\mathrm{CdS}$ using the successive ionic layer adsorption and reaction technique ${ }^{22}$ followed by high temperature annealing (Sec. II, SI). Xray diffraction confirmed the hexagonal structure for the doped as well as undoped CdS, (Fig. S1, SI) with no signature of any impurity phase. Transmission electron microscopy confirmed the uniform spherical morphology of both undoped and doped systems (Fig. S2, SI). The 
comparison of $\mathrm{Fe}$ and $\mathrm{Cd} \mathrm{K}$-edge in $\mathrm{Fe}$ doped $\mathrm{CdS}$ obtained by extended x-ray absorption fine structure (Fig, S3, SI) suggests that Fe is replacing Cd in the CdS lattice, corroborating the fact that $\mathrm{Fe}$ is acquiring a +2 oxidation state in the $\mathrm{CdS}$ lattice similar to earlier literature. ${ }^{23}, 24$ Further, the doped system is devoid of any impurity phase of the $\mathrm{Fe}_{3} \mathrm{O}_{4}$ core, as the $\mathrm{Fe} \mathrm{K}$-edge for the doped system is very different from the Fe K-edge for the core (Fig. S3, SI). Similar to earlier literature, ${ }^{23,24}$ no other oxidation state of $\mathrm{Fe}$ ions has been observed in our work in the final sample due to the extended annealing. The absorption spectra exhibit a bandgap of $\sim 2.5$ $\mathrm{eV}$ for both pristine and $\mathrm{Fe}^{2+}$ doped systems, as shown in Fig. S4, SI. TA spectroscopy ${ }^{25}$ (Sec. SIII, SI), both with linearly and circularly polarized light, was used to study the de-excitation pathways of the doped and pristine CdS. Non-resonant excitation was performed at $3.1 \mathrm{eV}$ photon energy, while resonant excitation was carried out at $2.58 \mathrm{eV}$.

For non-resonant excitation, the TA time trace for pristine CdS NCs at $2.5 \mathrm{eV}$ probe energy (shown in Fig. 1a) reveals a very long excited-state lifetime (> 1 ns measurement window, as shown in Fig. S5a, SI), demonstrating the high quality of NCs and the absence of surface trap states for the electron/hole. The positive differential transmission $(\Delta \mathrm{T} / \mathrm{T})$ signal is assigned to transient photobleaching $(\mathrm{PB})$ of the band-edge excitonic transition. The signal also shows a build-up time constant of $460 \pm 10$ fs (Fig. 1b), due to electron/hole relaxation from the higher energy excitonic states, followed by a biexponential decay, with a small amplitude component with a time constant of $4.85 \pm 0.2 \mathrm{ps}$ and a long-lived signal $>1000 \mathrm{ps.} \mathrm{In} \mathrm{contrast,} \mathrm{Fe}^{2+}$ doped CdS NCs reveal a dramatic difference in the dynamics, namely the PB signal decays within a few picoseconds as shown in Fig. 1a and the $\Delta$ T/T colour maps (Fig. S5b, SI). This difference is attributed to acceptor states created by $\mathrm{Fe}^{2+}$ doping. Early time data of $\mathrm{Fe}^{2+}$ doped $\mathrm{CdS}$ decay demonstrates that all concentrations of $\mathrm{Fe}^{2+}$ doping show an instantaneous rise and a very fast decay, as shown in Fig. 1b. The decay time constant of the PB signal of $\mathrm{Fe}^{2+}$ doped $\mathrm{CdS}$ decreases from $1.37 \pm 0.04$ ps to $312 \pm 10$ fs with an increasing percentage of Fe (Table S2, SI). This decay is similar to the rise time in undoped CdS, explaining the absence of band-edge PL in $\mathrm{Fe}^{2+}$ doped CdS. Importantly, the gradual and systematic decrease in the exciton lifetime of $\mathrm{Fe}^{2+}$ doped CdS with an increasing percentage of Fe (Table S2, SI) indicates the involvement of a density of states arising from the interaction of $\mathrm{Fe}^{2+}$ with the host rather than just one single acceptor state of Fe. Additionally, when the decay time-constant is longer than the rise time of pristine $\mathrm{CdS}$ exciton (for example, $1.37 \pm 0.04 \mathrm{ps}$ in $5 \% \mathrm{Fe}^{2+}$ doped $\mathrm{CdS}$ ), we do not observe a complete decay of the PB signal, suggesting that few of the excitons do not decay through the fast Fe channel.

To shed light on the electronic properties of pristine/ $\mathrm{Fe}^{2+}$ doped $\mathrm{CdS}$, we used the firstprinciples density functional theory (DFT) to determine the spin-resolved projected density of states (PDOS). Undoped CdS, being a non-magnetic system, exhibits degeneracy between the two spin states (Fig S6, SI). Conduction band minimum (CBM) of undoped CdS is primarily comprised of $\mathrm{Cd}-5 \mathrm{~s}$ orbitals while the valence band maximum (VBM) has major contributions from S-3p orbitals. DFT calculations with substitution of single and pairs of Fe ions in a $2 \times 2 \times 2$ supercell of CdS reveal Fe acceptor states. The negative formation energy of Fe substituted CdS (Sec. IV, SI) confirms the stability of substitution. Using Lowdin charge analysis it has been shown that the s-orbitals of Fe lose $\sim 2$ electrons, forming $\mathrm{Fe}^{2+}$ ions. There is not a 
significant change in the Lowdin charges of Fe going from ground state to excited state and hence the oxidation state remains +2 . (Sec. IV, SI). In its ground state, Fe states are mainly observed as spin-up states along with the S-3p states in the VBM while the CBM largely comprises the spin-down states at $\sim 1 \mathrm{eV}$ resulting in mid-gap states as evident in Fig. S7, SI due to the magnetic nature of Fe ion.

We modelled an exciton using the constrained occupation of electronic states to estimate the exciton formation energy (Sec. IV, SI). Since $\mathrm{Fe}^{2+}$ doped CdS is a magnetic system, excitation of a spin-up/down electron is not equivalent. We find that the excitation of a spin-up electron involves exciton formation energy of $0.70 \mathrm{eV}$, while that of a spin-down electron being 0.14 eV (Table S3, SI) with strictly enforced spin selection rules. To understand the interaction of $\mathrm{Fe}^{2+}$ with the host, we examine the PDOS and their respective charge density of frontier states in Fig. 1c-1f. For spin-down excitation, the acceptor state is delocalized within the host while the electron state is localized at the Fe ion, suggesting the decay through the Fe states to the host. In contrast, when the electron is excited from the spin-up states, both electron and hole are delocalized within the host with no involvement of the Fe states, suggesting a decay through the band-edge. This not only demonstrates the non-equivalence of the two spin states but also corroborates the fact that the exciton decay involves the host and hence depends on the density of states and not just on the atomic-like Fe levels. Further, calculation reveals a reduction in the magnetic moment of the $\mathrm{Fe}$ ion from its value of $3.25 \mu_{\mathrm{B}} / \mathrm{Fe}$ in the ground state to 2.56 $\mu_{\mathrm{B}} / \mathrm{Fe}$ in the spin-up and $3.00 \mu_{\mathrm{B}} / \mathrm{Fe}$ in the spin-down excited states, reflecting the different interaction of the spin states with the host material.

However, it is important to note that this strong hybridization of Fe with the valence band of the host is only true for low concentration of Fe ions, that is, within the regime, wherein a Fe atom would not see the presence of other Fe atoms. We study the role of $\mathrm{Fe}-\mathrm{Fe}$ interactions by substituting two Fe atoms/cell in our calculations. The relative positions of the two Fe-atoms were finalized using energy minimization calculations (DFT). PDOS and charge density of the lowest energy configuration with interacting Fe ions are summarized in Sec IV, SI. The lowest energy structure (Sec. IV, SI) shows an exciton formation energy of the spin-down electrons of $0.17 \mathrm{eV}$ that is much lower than the spin-up electrons $(1.59 \mathrm{eV})$. Furthermore, Fe-3d states behave as both donor and acceptor for the spin-down states, while the spin-up states are dominated by contributions from the $\mathrm{Cd} 5 \mathrm{~s}$ and $\mathrm{S} \mathrm{3p}$, with the same magnetic moment of 3.42 $\mu_{\mathrm{B}} / \mathrm{Fe}$ in both ground and excited states (Fig. S8, SI). Hence magneto-optical interaction of the magnetic ion with the host is destroyed and localized Fe acceptor states lead to instantaneous quenching of the excitonic states as observed in earlier literature due to intraionic transitions of magnetic dopants in semiconductors. ${ }^{26-28}$ Thus, clearly, the presence of Fe-Fe interactions is detrimental to the observed magneto-optical splitting of the spin states. 


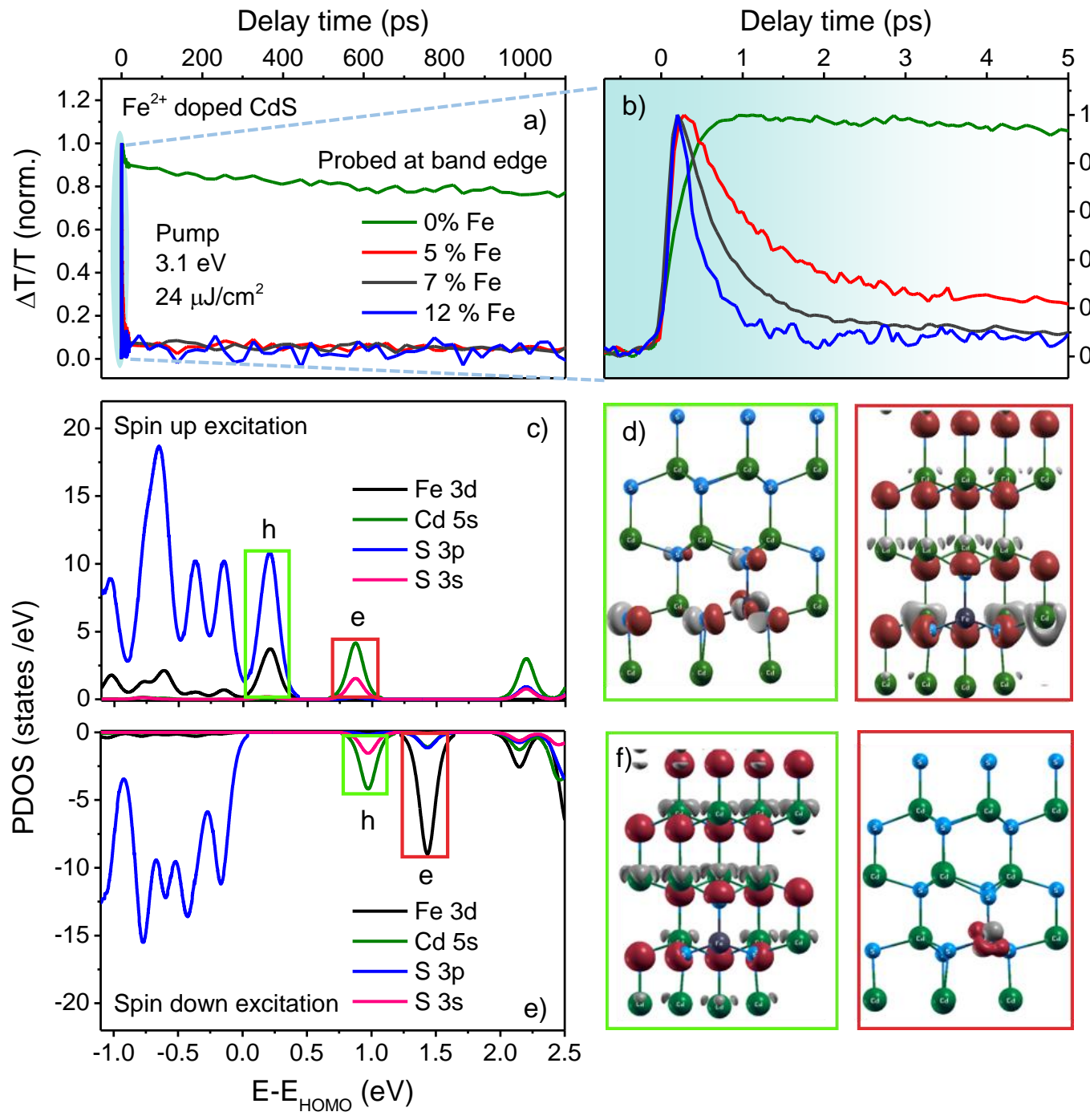

Figure 1. (a) Differential transmission dynamics of $\mathrm{Fe}^{2+}$ doped and undoped CdS NCs and zoom-in of the dynamics up to 5 ps region in (b). Spin-resolved projected density of states (PDOS) of (c) Spin-up excitation, (d) Charge density iso-surfaces depicting the spin-up excited state hole and electron position; (e) PDOS of spin-down excitation of $\mathrm{Fe}^{2+}$ doped CdS. (f) Charge density iso-surfaces depicting the spin-down excited state hole and electron position. Red and silver colours represent positive and negative charge density respectively. Green, grey and blue spheres correspond to $\mathrm{Cd}, \mathrm{Fe}$ and $\mathrm{S}$ atoms, respectively. Green and red open rectangles denote the hole and electron states, respectively.

We propose a simplified energy line diagram with the contributions from the various atomic orbitals to the spin-up/down excited states in Fig. $2 \mathrm{a} / 2 \mathrm{~b}$ respectively based on observations from the PDOS and the charge density iso-surfaces. The spin-up excitation is relatively unhindered as shown in Fig. 2a with regular photobleaching in TA spectra. However, the absorption is particularly relevant in the spin-down excitation as shown in Fig. $2 b$ wherein it exhibits a reduction below the resonance energy and an increase above the resonance energy leading to a nearly symmetrical derivative-like feature. The photoexcitation is just a small perturbation that initiates the magneto-optical splitting leading to MOSE that would however persist even beyond the duration of the excitation light pulse if the spin selection rules remain 
valid. Therefore, the excitation fluence required to achieve the currently reported splitting is also much lower $\left(24 \mu \mathrm{J} / \mathrm{cm}^{2}\right)$ than the optical Stark shifts in non-magnetic systems reported in the literature. Typical experimental signatures of a simple OSE include a transient shift of the optical absorption edge upon excitation with a non-resonant laser and a near symmetrical derivative-like feature in differential transmission spectra arising from the shift in energy levels. However, in this case, wherein the excitation is not spin-selective, we observe a sum of these effects along with normal PB as shown in Fig. 2c. The exact Stark shift for different Fe concentrations at different time intervals is calculated similar to literature ${ }^{1}$ and is discussed briefly in Sec V, SI and depicted in Fig. 2 d.
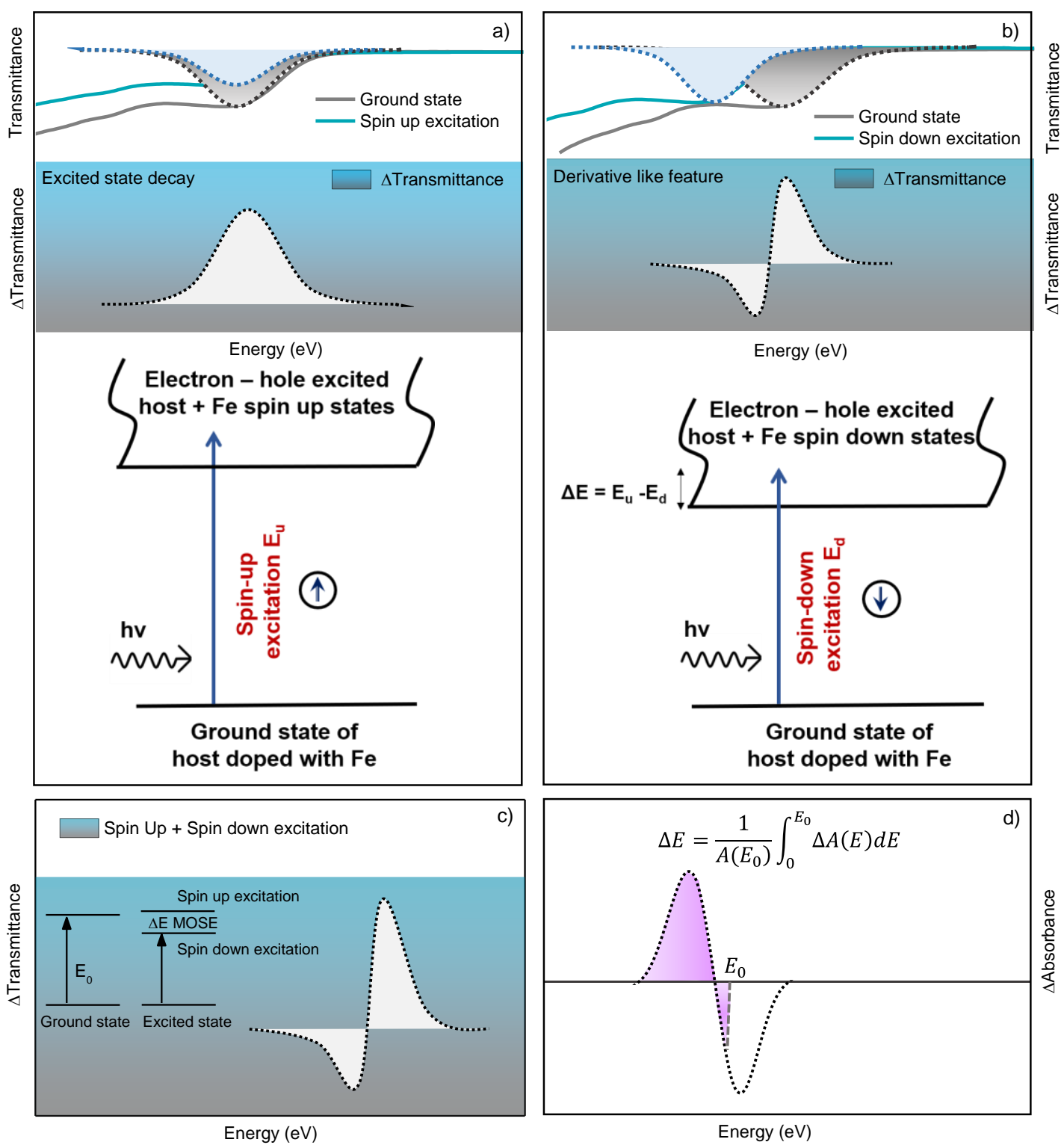

Figure 2. Illustration of (a) spin-up excitation and (b) spin-down excitation along with the corresponding line diagram showing the spin-up and spin-down excited states. Schematic representing (c) sum of spin-up and spin-down excitation, (d) Stark shift calculation.

Figures $3 \mathrm{a}$ and $3 \mathrm{~b}$ show the TA spectra at two different time delays of the NCs, under nonresonant excitation $(3.1 \mathrm{eV})$ using linearly polarised light. Undoped CdS NCs are characterized 
by a single PB peak centered around $2.49 \pm 0.01 \mathrm{eV} . \mathrm{Fe}^{2+}$ doped systems manifest instead typical Stark shift spectral signature, namely a derivative-like feature, characterized by a shifting $(\Delta \mathrm{E})$ of the excitonic absorption peak ( $\left.\mathrm{E}_{0}\right)$. This shift signifies the shift of energy levels, specifically near the VBM-CBM energies. It is important to note here that the energy splitting is quite small, and both the states would be accessible during the excitation at room temperature due to the intrinsic width of the laser excitation. However, the derivative-like difference spectrum suggests the presence of more than one pathway. One such pathway would be the conventional biexciton effect ${ }^{29-31}$ wherein a derivative-like signature stems from the overall sum of the PB signal and photoinduced absorption. However, we are working with very low fluence (24 $\mu \mathrm{J} / \mathrm{cm}^{2}$ ) corresponding to an average exciton $\langle\mathrm{N}\rangle$ of about 0.335 as shown in Table S6, SI. Additionally, our studies at other lower fluences showed identical data (Fig. S9, SI) suggesting that the biexciton effect is not responsible for these observations. We also note that in literature this derivative-like feature due to a biexciton effect is present only "immediately after excitation" $(<2 \mathrm{ps})$ at higher than band gap excitation. Further, this characteristic feature persists at all measured times after the excitation in the case of $\mathrm{Fe}^{2+}$ doped CdS NCs (Fig 3b), with a similar magnitude beyond the duration of the pulse but dependent on the $\mathrm{Fe}$ concentration in the host as shown in the inset to Fig. 3a. This splitting of the host spin states in presence of a magnetic ion like $\mathrm{Fe}$ is comparable to a Zeeman effect with a $\sim 2 \mathrm{~T}$ magnetic field at room temperature. On the other hand, the use of light as a perturbation without the magnetic ion would require an increase in the fluence by at least one order of magnitude, as observed from references in Table S1, SI. However, a combination of OSE with the internal perturbative field through the magneto-optical effects generates a spin-dependent quantumconfined Stark effect.

To obtain definitive proof of our proposed mechanism of spin-dependent magneto-optical effect within the excited state, we have performed helicity-resolved pump-probe measurements. These measurements were carried out using right circularly polarized probe pulses while the pump pulses were alternatively polarized with left or right circular polarization. Polarization-resolved spectra (Fig. 3c and Fig. S10a) and dynamics of $\Delta T / T$ (Fig. $\mathrm{S} 10 \mathrm{~b}$ ) are measured for $5 \% \mathrm{Fe}^{2+}$ doped $\mathrm{CdS}$ at $5.5 \mu \mathrm{J} / \mathrm{cm}^{2}$ pump fluence and $3.1 \mathrm{eV}$ pump energy with same and opposite helicities. The difference signal for the same and opposite circular polarisations of the pump and probe pulses is also shown in the figures, demonstrating a clear $\Delta \mathrm{T} / \mathrm{T}$ dependence on the circular polarization. This suggests the presence of two different states that show a change in the orientation of a component of the angular momentum of the system. This effect instead does not occur in a purely spin-independent wavepacket. ${ }^{1,32}$ Additionally, we also show that the difference TA spectrum is non-zero even up to the largest time scale of measurement (1200 ps) supporting our observation of long-lived spin-dependent optical phenomena (Fig. S10a, b). 

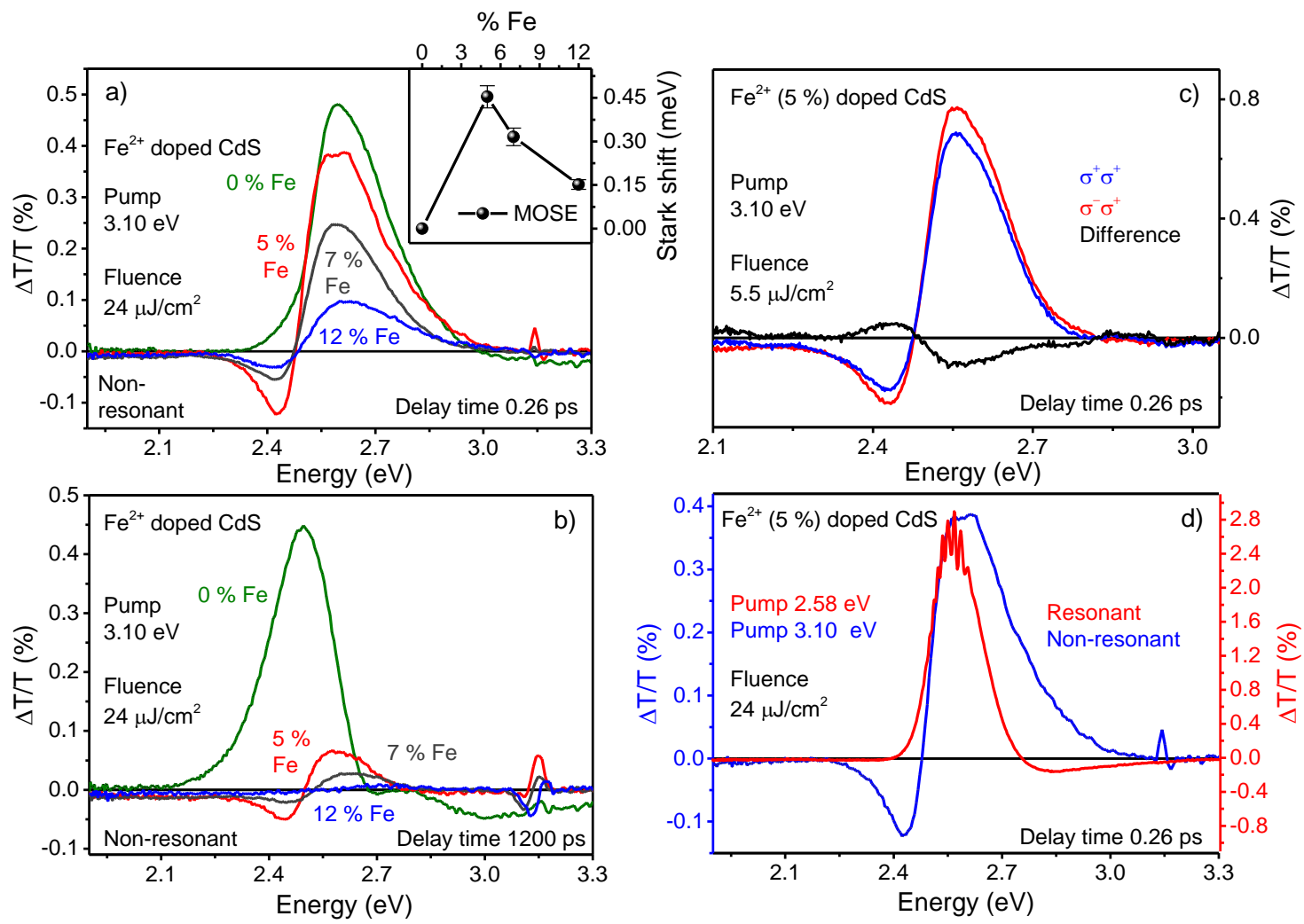

Figure 3. TA spectra of $\mathrm{Fe}^{2+}$ doped and undoped $\mathrm{CdS}$ shown at different pump-probe delay of $0.26 \mathrm{ps}$ (a) and $1200 \mathrm{ps}$ (b), for a pump photon energy of $3.1 \mathrm{eV}$ which is above the exciton resonance energy. Magnitude of Stark shifts (with error bars) in the inset to (a). (c) TA spectra of $\mathrm{Fe}^{2+}(5 \%)$ doped $\mathrm{CdS}$ using same and opposite pump-probe circular polarizations shown at a pump-probe delay of $0.26 \mathrm{ps}$, along with the difference spectrum (d) TA spectra of $\mathrm{Fe}^{2+}(5 \%)$ doped $\mathrm{CdS}$ under resonant and non-resonant excitations at a pump-probe delay of $0.26 \mathrm{ps}$.

Further, we have studied the fluence dependence of magneto-optical splitting. Even with the lowest fluence used $\left(2.7 \mu \mathrm{J} / \mathrm{cm}^{2}\right)$, the derivative signal persists for extended time scales supporting the occurrence of MOSE (Fig. S11, SI). Further, to ensure that these observed signatures correspond to the Stark shift, we performed linearly polarized pump-probe measurements under resonant conditions wherein an energy splitting rather than a Stark shift should be present. We expect a decrease in transmission at energies both above and below the resonance and an increase in transmission at resonance. This typical response is indeed shown in Fig. 3d (red line) in contrast to the Stark shift in the non-resonant excitation (blue line).

In summary, we demonstrated the MOSE by studying the magneto-optical interactions of the dopant states with the host electronic states in $\mathrm{Fe}^{2+}$ doped $\mathrm{CdS} \mathrm{NCs}$, in the absence of Fe-Fe interactions. Linearly and circularly polarized TA spectroscopy and DFT calculations were combined to understand the electron transfer within the host into Fe-3d states, elucidating the quenching behaviour as well as the Stark shift. We showed the emergence of MOSE due to the magneto-optical interaction for an extended amount of time at room temperature. DFT calculations of transient and ground-state electronic structure and properties also mirrored these changes in the transition. 


\section{AUTHOR INFORMATION}

Corresponding author

*E-mail: rv@jncasr.ac.in

Competing interests: The authors declare no competing interests.

Supporting Information: Synthesis of $\mathrm{Fe}^{2+}$ doped and undoped CdS NCs, TA measurement details, DFT calculation details, XRD, TEM, EXAFS spectra, linear absorption spectra, $\Delta \mathrm{T} / \mathrm{T}$ maps, spin-resolved projected DOS for $\mathrm{Fe}^{2+}$ doped and undoped $\mathrm{CdS}$, circularly polarized TA spectra and dynamics for $\mathrm{Fe}^{2+}$ doped $\mathrm{CdS}$, estimation of MOSE.

\section{$\underline{\text { Acknowledgment }}$}

The authors thank JNCASR, Sheikh Saqr Laboratory, Science and Engineering Research Board, Department of Science and Technology, Government of India, for financial support. S.G. and G.C. acknowledge support from the European Commission through the Marie Sklodowska-Curie actions (project CHIRALSCOPY, H2020- MSCA-IF-2018-841356).

\section{Reference:}

1. Giovanni, D.; Chong, W. K.; Dewi, H. A.; Thirumal, K.; Neogi, I.; Ramesh, R.; Mhaisalkar, S.; Mathews, N.; Sum, T. C. Tunable room-temperature spin-selective optical Stark effect in solution-processed layered halide perovskites. Sci. Adv. 2016, 2, (6), e1600477. 2. Amo, A.; Liew, T. C. H.; Adrados, C.; Houdré, R.; Giacobino, E.; Kavokin, A. V.; Bramati, A. Exciton-polariton spin switches. Nat. Photonics 2010, 4, (6), 361-366.

3. Reiter, D. E.; Axt, V. M.; Kuhn, T. Optical signals of spin switching using the optical Stark effect in a Mn-doped quantum dot. Phys. Rev. B 2013, 87, (11), 115430.

4. $\quad$ Wolf, S. A.; Awschalom, D. D.; Buhrman, R. A.; Daughton, J. M.; von Molnár, v. S.; Roukes, M. L.; Chtchelkanova, A. Y.; Treger, D. M. Spintronics: a spin-based electronics vision for the future. Science 2001, 294, (5546), 1488-1495.

5. Udo, M. K.; Villeret, M.; Miotkowski, I.; Mayur, A. J.; Ramdas, A. K.; Rodriguez, S. Electronic excitations of substitutional transition-metal ions in II-VI semiconductors: CdTe: $\mathrm{Fe}^{2+}$ and CdSe: $\mathrm{Fe}^{2+}$. Phys. Rev. B 1992, 46, (12), 7459-7468.

6. Vallin, J. T.; Slack, G. A.; Bradley, C. C. Far-infrared absorption of $\mathrm{ZnS}: \mathrm{Fe}^{2+}$ in strong magnetic fields. Phys. Rev. B 1970, 2, (11), 4406-4413.

7. Smoleński, T.; Kazimierczuk, T.; Kobak, J.; Goryca, M.; Golnik, A.; Kossacki, P.; Pacuski, W. Magnetic ground state of an individual $\mathrm{Fe}^{2+}$ ion in strained semiconductor nanostructure. Nat. Commun. 2016, 7, (1), 10484.

8. Xia, Y.; Li, Q.; Kim, J.; Bao, W.; Gong, C.; Yang, S.; Wang, Y.; Zhang, X. Roomtemperature giant Stark effect of single photon emitter in van der Waals material. Nano Lett. 2019, 19, (10), 7100-7105.

9. Perebeinos, V.; Avouris, P. Exciton ionization, Franz- Keldysh, and Stark effects in carbon nanotubes. Nano Lett. 2007, 7, (3), 609-613.

10. Yang, Y.; Yang, M.; Zhu, K.; Johnson, J. C.; Berry, J. J.; Van De Lagemaat, J.; Beard, M. C. Large polarization-dependent exciton optical Stark effect in lead iodide perovskites. Nat. Commun. 2016, 7, (1), 12613.

11. Joffre, M.; Hulin, D.; Migus, A.; Combescot, M. Laser-induced exciton splitting. Phys. Rev. Lett. 1989, 62, (1), 74-77. 
12. Stern, L.; Desiatov, B.; Mazurski, N.; Levy, U. Strong coupling and high-contrast alloptical modulation in atomic cladding waveguides. Nat. Commun. 2017, 8, (1), 14461.

13. Liu, M.; Yin, X.; Ulin-Avila, E.; Geng, B.; Zentgraf, T.; Ju, L.; Wang, F.; Zhang, X. A graphene-based broadband optical modulator. Nature 2011, 474, (7349), 64-67.

14. Bludau, W.; Wagner, E. Impact ionization of excitons in GaAs. Phys. Rev. B 1976, 13, (12), 5410-5414.

15. Empedocles, S. A.; Bawendi, M. G. Quantum-confined Stark effect in single CdSe nanocrystallite quantum dots. Science 1997, 278, (5346), 2114-2117.

16. Walters, G.; Wei, M.; Voznyy, O.; Quintero-Bermudez, R.; Kiani, A.; Smilgies, D. M.; Munir, R.; Amassian, A.; Hoogland, S.; Sargent, E. The quantum-confined Stark effect in layered hybrid perovskites mediated by orientational polarizability of confined dipoles. Nat. Commun. 2018, 9, (1), 4214.

17. Yuhas, B. D.; Fakra, S.; Marcus, M. A.; Yang, P. Probing the local coordination environment for transition metal dopants in zinc oxide nanowires. Nano Lett. 2007, 7, (4), 905909.

18. Segura-Ruiz, J.; Martinez-Criado, G.; Chu, M.; Geburt, S.; Ronning, C. Nano-X-ray absorption spectroscopy of single Co-implanted $\mathrm{ZnO}$ nanowires. Nano Lett. 2011, 11, (12), $5322-5326$.

19. Keavney, D. J.; Cheung, S. H.; King, S. T.; Weinert, M.; Li, L. Role of defect sites and Ga polarization in the magnetism of Mn-doped GaN. Phys. Rev. Lett. 2005, 95, (25), 257201.

20. Saha, A.; Makkar, M.; Shetty, A.; Gahlot, K.; Pavan, A. R.; Viswanatha, R. Diffusion doping in quantum dots: bond strength and diffusivity. Nanoscale 2017, 9, (8), 2806-2813.

21. Makkar, M.; Viswanatha, R. Frontier challenges in doping quantum dots: synthesis and characterization. $R S C A d v$. 2018, 8, (39), 22103-22112.

22. Valkonen, M. P.; Kanniainen, T.; Lindroos, S.; Leskelä, M.; Rauhala, E. Growth of $\mathrm{ZnS}, \mathrm{CdS}$ and multilayer ZnS/CdS thin films by SILAR technique. Appl. Surf. Sci. 1997, 115, (4), 386-392.

23. Saha, A.; Shetty, A.; Pavan, A.; Chattopadhyay, S.; Shibata, T.; Viswanatha, R. Uniform doping in quantum-dots-based dilute magnetic semiconductor. J. Phys. Chem. Lett. 2016, 7, (13), 2420-2428.

24. Saha, A.; Chattopadhyay, S.; Shibata, T.; Viswanatha, R. Core-Shell to Doped Quantum Dots: Evolution of the Local Environment Using XAFS. J. Phys. Chem. C 2016, 120, (33), 18945-18951.

25. Maiuri, M.; Garavelli, M.; Cerullo, G. Ultrafast spectroscopy: state of the art and open challenges. J. Am. Chem. Soc. 2020, 142, 3-15.

26. Zanella, M.; Falqui, A.; Kudera, S.; Manna, L.; Casula, M. F.; Parak, W. J. Growth of colloidal nanoparticles of group II-VI and IV-VI semiconductors on top of magnetic ironplatinum nanocrystals. J. Mater. Chem. 2008, 18, (36), 4311-4317.

27. Mirov, S.; Fedorov, V.; Moskalev, I.; Mirov, M.; Martyshkin, D. Frontiers of midinfrared lasers based on transition metal doped II-VI semiconductors. J. Lumin. 2013, 133, 268-275.

28. Piotrowski, P.; Pacuski, W. Photoluminescence of CdTe quantum wells doped with cobalt and iron. J. Lumin. 2020, 221, 117047.

29. Makarov, N. S.; Guo, S.; Isaienko, O.; Liu, W.; Robel, I.; Klimov, V. I. Spectral and dynamical properties of single excitons, biexcitons, and trions in cesium-lead-halide perovskite quantum dots. Nano Lett. 2016, 16, (4), 2349-2362.

30. Kambhampati, P. Hot exciton relaxation dynamics in semiconductor quantum dots: radiationless transitions on the nanoscale. J. Phys. Chem. C 2011, 115, (45), 22089-22109. 
31. Yumoto, G.; Tahara, H.; Kawawaki, T.; Saruyama, M.; Sato, R.; Teranishi, T.; Kanemitsu, Y. Hot biexciton effect on optical gain in $\mathrm{CsPbI}_{3}$ perovskite nanocrystals. J. Phys. Chem. Lett. 2018, 9, (9), 2222-2228.

32. Sokell, E.; Zamith, S.; Bouchene, M. A.; Girard, B. Polarization-dependent pumpprobe studies in atomic fine-structure levels: towards the production of spin-polarized electrons. J. Phys. B: At. Mol. Opt. Phys. 2000, 33, (11), 2005.

TOC Graphic

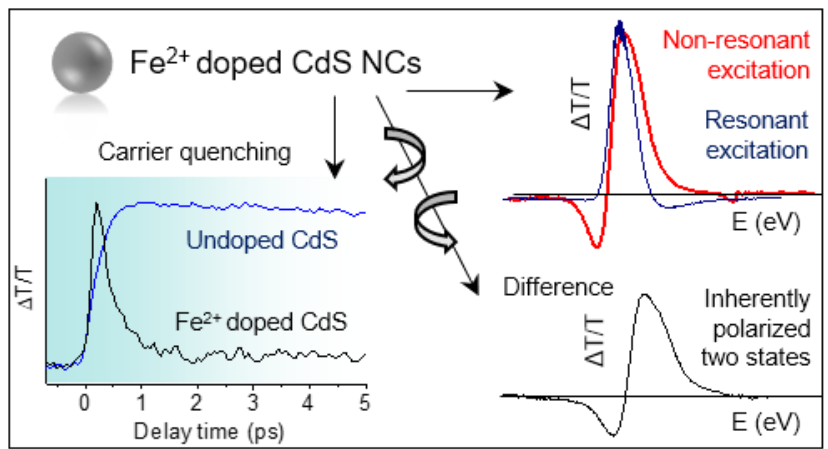

